\title{
Studies about an Equation of State for Pure Associated Fluids: Temperature Dependent Co-Volume Accounting a Physically Consistent Repulsive Term
}

\author{
R. F. Checoni* ${ }^{1}$ and S. P. Ravagnani ${ }^{2}$ \\ ${ }^{1}$ Chemical Engineering Program - Texas A\&M University at Qatar - Doha - Qatar \\ ${ }^{2}$ Faculdade de Engenharia Química, Universidade Estadual de Campinas - UNICAMP - Brazil. \\ E-mail: ${ }^{1}$ rfcheconi@yahoo.com.br
}

\begin{abstract}
Studies related to the development of equations of state (EOS) to represent thermophysical properties of pure compounds are considered as important tools for engineers to design and optimize industrial equipment and processes. Furthermore, these tools also contribute to amplify the researchers' knowledge related to molecular interaction types, in attempting to predict and correlate both energetic and volumetric effects existing in the compounds. From several equations of state existing, the cubic plus association (CPA) EOS are employed in the calculations of thermophysical properties of compounds, in which the molecular interactions occurring are the association type. In spite of good representation of these properties, it is possible to improve the predictive and correlative capability of the CPA EOS by substitution of terms whose physical meaning can be better. In this way, modifications of the cubic plus association equation of state are proposed: the original repulsive term is replaced by the Carnahan-Starling repulsion term; the attractive term is changed to an attraction term similar to the PengRobinson EOS. Furthermore, both attraction and repulsion terms are taken to be temperature dependent when alpha and beta functions are employed in calculations. All implementations make the equations of state non-cubic in relation to volume. Vapor pressure and liquid molar volumes of 1-alkanols $\left(C_{1}\right.$ to $\left.C_{10}\right)$ and water were correlated to experimental data using this non-CPA-EOS format, and good agreement is observed.
\end{abstract}

Keywords: CPA-EOS; equation of state; generalized alpha and beta functions; modeling; pure compounds.

\section{Introduction}

Studies related to the development of equations of state (EOS) to represent adequately the thermodynamic behavior of pure compounds are considered necessary tools to design and optimize equipment and industrial processes. Besides, the results generated by EOSs contribute also to researchers' knowledge about the interactions existing between molecules. Before proposing an equation of state, it is necessary to take in to account some conditions such as the type of compound studied (non-polar or polar), and the range conditions where the EOS work efficiently (melting to critical conditions or supercritical conditions). Another important condition that makes an equation of state useful is the generalization of its adjustable parameters to an ever larger number of pure components; this practice is common in EOSs studies to help us to enhance the scope of application.

Based on these necessities and considerations several equations of state have been proposed: van der Waals (Abbott, 1989), Soave-Redlich-Kwong (SRK) (Soave, 1972), and Peng-Robinson (PR) (Peng \& Robinson, (1976)) can be cited as examples; due to their simplicity, these EOSs became the most used in the industrial and research applications. Moreover, several modifications have been proposed (Wei \& Sadus, 2000) in an attempt to improve their predictive capability of the phase equilibria behavior of fluids, for both pure compounds and mixtures. However, equations of state in their original format are not adequate to predict thermodynamic behavior of associating species such as water or alcohols that exhibit hydrogen-bonding interaction types. Nevertheless, special attention is given to a specific group of EOSs: those accounting for the hydrogen bond effects existing in associating species. To this group belongs the statistical associating fluid theory (SAFT) (Huang \& Radosz, 1990) EOS and cubic plus association CPA (Kontogeorgios et al., 1996) EOS. The first one combines two terms: one related to the contribution of the physical forces and other for the contribution of association (hydrogen-bonds); the second combines a cubic EOS (SRK) with the association part of the SAFT EOS. Using CPA EOS the computational time is reduced while its predicting accuracy is similar to that of the SAFT EOS.

Originally, the CPA EOS was proposed to correlate the vapor pressure and the saturated liquid molar volume of pure associating fluids (Kontogeorgios et al., 1996) (only some alcohols were studied). Subsequently, several studies involving other pure compounds and mixtures (as electrolytes, hydrocarbons, and water) have been realized to validate it, according to (Kontogeorgis et al., 2006); besides, experimental data of surface tension, solid-liquid equilibria, liquid-liquid equilibria, vapor-liquid equilibria, have been employed for correlative and predictive calculations. The CPA EOS also can be applied in the prediction of the monomeric fraction of pure compounds and mixtures of associating fluids (von Solms et al., 2006) and, by gradient theory combination, it is capable to model the vapor-liquid interfaces of aqueous + hydrocarbon mixtures, presenting high accuracy in the obtained results (Queimada et al., 2006). Both the vast application field and versatility of the CPA EOS is featured by the proposed modifications that contributed to improve the correlative 
and predictive capability of the thermophysical properties of both pure compounds and mixtures (Kontogeorgis et al., 2006).

The versatility demonstration can motivate changes in the CPA EOS in attempt to improve its physical consistency, even though the results of predicted properties are approximately similar to those obtained in the original versions of the CPA and SAFT equations of state. One of these changes can be attributed to the substitution of the repulsive term, in the cubic part of the CPA EOS, by one whose physical meaning is better. In spite of the existence of various options (Wei \& Sadus, 2000), the repulsive term employed in present work is that proposed by Carnahan and Starling (Carnahan \& Starling, 1969 \& 1972); this choice is based on the good predictive/correlative results presented by several works in the literature accounting this term in the equations of state (De Santis et al., 1976, Zhong \& Masuoka, 1977, Wei et al., 1996, Kutney et al., 1997, Sadus, 2001).

Another important advantage of an equation of state is the possibility to apply the same adjustable parameters set to various pure compounds, accounting a same conditions range. (It is noteworthy that either equation of state modification is firstly proposed for properties calculations of pure compounds since it is necessary, in most cases, to use the parameters of pure compounds to obtain information about the mixtures behavior). This feature cannot be observed in the original CPA EOS (Kontogeorgios et al., 1996) since each pure compound studied has its own adjustable parameter set. Some studies involving cubic equations of state suggest that it is possible to generalize the adjustable parameters of the various pure compounds (Toghiani \& Viswanath, 1986) and Xu \& Sandler, 1987). It is common for this generalization to account for mathematical expressions, known as alphafunction, whose relation is established with the temperature and acentric factor of the pure compounds; some examples of alpha functions can be found in (Mathias \& Copeman, 1983), (Trebble \& Bishnoi, 1987), and (Coquelet et al., 2004)

In addition to taking into account physicallly consistent term proposed by Carnahan and Starling (Carnahan \& Starling, 1969 \& 1972), and the generalization of the adjustable parameters, the present work also considers the co-volume parameter, as well the energetic parameter, temperature-dependent; this dependency is associated to the generalized $\alpha(\operatorname{Tr}, \omega)$ and $\beta(\operatorname{Tr}, \omega)$ functions. The attractive term of the equation of state cubic part is that present in the (Peng \& Robinson, 1976). The correlative capability using this proposed non-cubic plus associating EOS for vapor pressure and vapor and liquid molar volumes (Mathias \& Copeman, 1983), (Trebble \& Bishnoi, 1987), and (Coquelet et al., 2004) are analyzed, and comparisons with results obtained from associating equations of state are presented.

\section{Equations of State}

\subsection{The CPA Equation of State}

The original cubic plus associating equation of state combines the SRK EOS (Soave, 1972) with the association term (from Wertheim theory (Wertheim, $1984 \mathrm{a}$ and b), (Wertheim, $1986 \mathrm{a}, \mathrm{b}$, and c), and (Wertheim, 1987). The CPA EOS can be represented in terms of pressure by

$$
\begin{aligned}
p & =\frac{R T}{(V-b)}-\frac{a(T)}{V(V+b)} \\
& -0.5 \frac{R T}{V}\left[1+\rho \frac{\partial \ln g(\rho)}{\partial \rho}\right] \sum_{A}\left(1-X_{A}\right)
\end{aligned}
$$

in which $R$ is the universal gas constant, $V$ is the molar volume of the system, $b$ is the co-volume parameter (independent of the temperature) and $a(T)$ represents the attraction parameter, with temperature $(T)$ dependence. $X_{A}$ is the fraction of the A-sites that are not bonded with other sites. A simplified radial distribution function $g(\rho)$ version, already used in (Kontogeorgis et al., 1999), is represented by

$$
g(\rho)=\frac{1}{1-1.9 \eta}
$$

in which $\eta=b /(4 V)$, is the packing fraction of hard spheres. The site fraction $X_{A}$ is defined by

$$
X_{A}=\frac{1}{1+\rho \sum_{B} X_{B} \Delta^{A B}}
$$

in which the association strength $\Delta^{A B}$ between the sites $\mathrm{A}$ and $\mathrm{B}$ is represented by

$$
\Delta^{A B}=g(\rho)\left[\exp \frac{\varepsilon^{A B}}{R T}-1\right] b \kappa^{A B}
$$

wherein $\varepsilon^{A B}$ and $\kappa^{A B}$ are the energy of association and volume of association, respectively, existing between sites $A$ and $B$. The choice of the association scheme between molecules is an important decision that must be made, whose dependence must be related to the compounds studied. With the association scheme defined it is possible to determine the monomer fraction $X_{A}$. Both schemes and expressions are shown in (Huang \& Radosz, 1990).

\subsection{Carnahan-Starling Hard-Sphere Repulsion Term}

Carnahan and Starling (Carnahan \& Starling, 1969 \& 1972) developed an equation of state for non-attracting hard-spheres based on an analysis of the reduced geometric series of the virial equation. The expression is

$$
p=\frac{R T\left(1+y+y^{2}-y^{3}\right)}{V(1-y)^{3}}
$$

in which $y=b /(4 V)$. This form is the most widely used alternative to the van der Waals hard-sphere term due to comparing very well with molecular simulation data (Mathias \& Copeman, 1983) for phase equilibria. Using the ideas of (Carnahan-Starling, 1969 \& 1972), various researchers proposed equations of state that incorporate the Eq. (5): (De Santis et al., 1976, Zhong \& Masuoka, 1977, Wei et al., 1996, Kutney et al., 1997, Sadus, 2001).

\subsection{Generalized Alpha Function $\alpha(T r)$}

Accurate representation of pure compound vapor pressures by equations of state is important in phase equilibria calculations. In these equations, adjustable 
parameters are obtained empirically using temperature dependence established with equilibrium experimental data. In EOSs such as SRK and PR, for example, this dependency is accounted for only in the attraction parameter $a(T)$ by means of a characteristic expression (known as alpha function $\alpha(T r)$ ). In an attempt to improve the prediction or correlation of the phase equilibria, several alpha functions have been proposed (Soave, 1972), (Peng \& Robinson, 1976), (Toghiani \& Viswanath, 1986), Xu \& Sandler, 1987), and (Mathias \& Copeman, 1983). Generally, the alpha functions are developed in terms of reduced temperature, which is adequate to the group of substances studied in the development of each $\alpha(T r)$. The generalization in terms of the pure compounds set is usual, and its characterization is made considering the Pitzer acentric factor, which is specific for each pure compound, as presented in (Peng \& Robinson, 1976), (Wei \& Sadus, 2000), (Mathias \& Copeman, 1983), (Trebble \& Bishnoi, 1987), and (Coquelet, et al., 2004).

Another way to improve phase equilibria representation considers the co-volume parameter (represented by $b$ ) to be temperature dependent. Fuller (Fuller, 1976), (Haman et al., 1977), Ravagnani and D'Ávila (Ravagnani \& D'Avila, 1985), and Toghiani and Viswanath (Toghiani \& Viswanath, 1986) proposed modifications of the SRK EOS considering the $b$ parameter temperature dependent; (Xu \& Sandler, 1987), (Nasrifar \& Moshfeghian, 2001), and (Haghtalab et al. 2011) proposed the $b$ parameter to be temperature dependent for the PR EOS. All work showed that the agreement between experimental data and those calculated from equations of state was enhanced with $b(T r)$.

\subsection{Modification in CPA EOS}

Replacing the SRK EOS repulsive and attractive terms of original CPA-EOS, Eq. (1), by the hard-sphere repulsion term proposed by Carnahan-Starling (Carnahan \& Starling, $1969 \&$ 1972), and by the original Peng-Robinson EOS (Peng \& Robinson, 1976), respectively, and taking into account the temperature dependence of the co-volume parameter, it is possible to obtain the following non-cubic expression in terms of the molar volume:

$$
\begin{aligned}
p_{\text {non-cubic }}= & \frac{R T\left(1+y+y^{2}-y^{3}\right)}{V(1-y)^{3}} \\
& -\frac{a(T)}{V^{2}+2 V b(T)-b^{2}(T)}
\end{aligned}
$$

in which $y=b(T) /(4 V)$. The association term is the same as in the original CPA EOS, i.e.:

$$
p_{\text {Assoc }}=-0.5 \frac{R T}{V}\left[1+\rho \frac{\partial \ln g(\rho)}{\partial \rho}\right] \sum_{A}\left(1-X_{A}\right)
$$

Both attraction energetic and co-volume parameters are considered temperature dependent and are defined by the expressions

$$
\begin{aligned}
& a(T)=a_{\mathrm{c}} \alpha(T r) \\
& b(T)=b_{\mathrm{c}} \beta(T r)
\end{aligned}
$$

wherein the subscripts c indicate critical condition, and

$a_{\mathrm{c}}=0.51098 \frac{R^{2} T_{\mathrm{c}}^{2}}{p_{\mathrm{c}}}$

$b_{\mathrm{c}}=0.09764 \frac{R T_{\mathrm{c}}}{p_{\mathrm{c}}}$

The two expressions above as well as the $\alpha(T r)$ and $\beta(T r)$ functions

$\alpha(\operatorname{Tr})=\left[1+P\left(1-\operatorname{Tr}^{0.5}\right)^{0.5}\right]^{2}$

and

$\beta(\operatorname{Tr})=\left[1+Q\left(1-\operatorname{Tr}^{0.5}\right)^{0.5}\right]^{2}$

are given elsewhere (Coquelet et al., 2004); in the present work, the studied $T r$ range was 0.5 to 1.0 . It is possible to observe that each function has an adjustable parameter $(P$ and $Q$ ) which are structured as power series, truncated at $2^{\text {nd }}$ order, in the acentric factor $(\omega)$ :

$P(\omega)=P_{1}+P_{2} \omega+P_{3} \omega^{2}$

$Q(\omega)=Q_{1}+Q_{2} \omega+Q_{3} \omega^{2}$

In the association part (Eq. (7)), the radial distribution function is represented by

$g(\rho)=\frac{4}{4-1.9 \rho b(T)}$

This expression is based on molecular density (temperaturedependent) instead of using the segment-diameter and segment-number (as in the SAFT EOS). It is obvious that this feature makes the proposed EOS simpler for calculations.

The pure compounds studied in the present work are 1alkanols and water. In this way, the association scheme adopted for both compounds is 3B (three sites), which is represented by $X_{A}=X_{B}$ and $X_{C}=2 X_{A}-1$, since this is the most adequate scheme for association studied by Perakis et al. (Perakis et al., 2006). The summation in the Eq. (7) is

$\sum_{A}\left(1-X_{A}\right)=4-4 X_{A}$

and the expression for $X_{A}$ is

$X_{A}=\frac{-\left(1-\rho \Delta^{A B}\right)+\left[\left(1+\rho \Delta^{A B}\right)^{2}+4 \rho \Delta^{A B}\right]^{0.5}}{4 \rho \Delta^{A B}}$

in which the association strength $\Delta^{A B}$ between the sites $A$ and $B$ is represented by

$\Delta^{A B}=\frac{4}{4-1.9 \rho b(T)}\left[\exp \frac{\varepsilon^{A B}}{R T}-1\right] b(T) \kappa^{A B}$ 
Table 1. Thermophysical properties and their average absolute deviation (AAD) for each pure compound.

\begin{tabular}{|c|c|c|c|c|c|c|c|c|c|}
\hline & $\begin{array}{c}P c \\
\text { (bar) }\end{array}$ & $\begin{array}{l}T c \\
(\mathbf{K})\end{array}$ & $\omega$ & $\boldsymbol{P}$ & $Q$ & $\varepsilon_{\left(\text {bar. L } . \mathrm{mol}^{-1}\right)}^{A B}$ & $\kappa^{A B}$ & $\begin{array}{c}\Delta P^{(*)} \\
(\%)\end{array}$ & $\begin{array}{c}\Delta V^{(*)} \\
(\%)\end{array}$ \\
\hline methanol & 78.817 & 512.64 & 0.5643 & 10.00 & 1.00 & 273.98 & 0.0110 & $0.79^{(18)}$ & $0.56^{(18)}$ \\
\hline ethanol & 59.776 & 514.07 & 0.6436 & 9.98 & 1.01 & 268.14 & 0.0093 & $0.59^{(15)}$ & $0.23^{(15)}$ \\
\hline 1-propanol & 50.354 & 536.71 & 0.6240 & 9.99 & 1.10 & 270.44 & 0.0110 & $0.55^{(21)}$ & $0.40^{(21)}$ \\
\hline 2-propanol & 46.385 & 508.45 & 0.6648 & 9.99 & 1.04 & 269.05 & 0.0161 & $0.60^{(17)}$ & $0.96^{(17)}$ \\
\hline 1-butanol & 43.081 & 563.20 & 0.5901 & 9.80 & 1.37 & 270.96 & 0.0110 & $0.56^{(18)}$ & $0.69^{(18)}$ \\
\hline 2-butanol & 40.705 & 536.05 & 0.5743 & 10.00 & 1.00 & 270.30 & 0.0126 & $0.54^{(18)}$ & $1.03^{(18)}$ \\
\hline 2-methyl-1-propanol & 41.335 & 547.93 & 0.5902 & 10.00 & 1.10 & 271.69 & 0.0110 & $0.68^{(12)}$ & $1.10^{(12)}$ \\
\hline 2-methyl-2-propanol & 38.192 & 506.36 & 0.6130 & 10.00 & 1.00 & 271.47 & 0.0110 & $1.07^{(12)}$ & $0.54^{(12)}$ \\
\hline 1-pentanol & 37.577 & 588.30 & 0.5785 & 9.61 & 1.15 & 271.18 & 0.0132 & $0.21^{(17)}$ & $0.92^{(17)}$ \\
\hline 1-hexanol & 39.447 & 611.00 & 0.5600 & 10.00 & 1.23 & 272.30 & 0.0254 & $0.18^{(19)}$ & $0.86^{(19)}$ \\
\hline 1-heptanol & 29.608 & 633.00 & 0.5920 & 10.00 & 0.95 & 273.10 & 0.0110 & $0.29^{(13)}$ & $0.92^{(13)}$ \\
\hline 1-octanol & 27.857 & 652.50 & 0.5870 & 9.98 & 1.52 & 269.77 & 0.0117 & $1.38^{(12)}$ & $0.98^{(12)}$ \\
\hline 1-nonanol & 22.086 & 671.00 & 0.5436 & 8.98 & 1.20 & 270.00 & 0.0599 & $0.03^{(14)}$ & $0.43^{(14)}$ \\
\hline 1-decanol & 21.623 & 687.00 & 0.6098 & 7.32 & 1.03 & 269.77 & 0.0004 & $1.45^{(12)}$ & $0.55^{(12)}$ \\
\hline water & 218.252 & 647.37 & 0.3480 & 0.074 & 1.15 & 268.11 & 0.0571 & $1.45^{(40)}$ & $0.86^{(40)}$ \\
\hline \multicolumn{8}{|c|}{ OVERALL DEVIATION (OD) } & $0.69^{(258)}$ & $0.74^{(258}$ \\
\hline
\end{tabular}

(*) The values in the parenthesis are refer to the number of data points.

Substituting Eqs. (16), (17), and (18) into the Eq. (7), the association contribution of the new EOS is

$$
\begin{aligned}
& p_{\text {Assoc }}=-2 \frac{R T}{V}\left[1+\rho \frac{1.9 b(T)}{4-1.9 \rho b(T)}\right] \\
&\left\{1-\left[\frac{\rho \Delta^{A B}-1+\left(\left(1+\rho \Delta^{A B}\right)^{2}+4 \rho \Delta^{A B}\right)^{0.5}}{4 \rho \Delta^{A B}}\right]\right\}
\end{aligned}
$$

\section{Results and Discussion}

The $a(T), b(T)$, and $\Delta^{A B}$ values in Eqs. (6) and (20) were obtained using the PVT experimental data (Beaton et al., $1989 \mathrm{a}$ and $\mathrm{b}$ ) for the alcohols and water, applying the Rosenbrock method (Rosenbrock, 1960) of sequential search, whose calculation routine is described in (Kuester \& Mize, (1973)); for calculation execution, a program in FORTRAN language was developed. The routine objective is to minimize the difference between the calculated $\left(P_{\text {calc }}\right)$ and experimental data $\left(P_{\exp }\right)$ of vapor pressure, using PVT data at various conditions $(0.50<T r \leq 1.00)$, to values lower than $10^{-4}$ bar. Subsequently, the alpha and beta values (Eqs. (8) and (9)) as well as $\left(\varepsilon^{A B}\right)$ and $\left(\kappa^{A B}\right)$, Eq. (19), were calculated for each pure compound.

For the determination of the $P$ and $Q$ adjustable parameters, the same Rosenbrock-Hillclimb routines were used, now minimizing (to values lower than $10^{-5}$ ) the difference between the alpha and beta values calculated in Eqs. (8) and (9) and those obtained from Eqs. (12) and (13), with a $T r$ range from 0.5 to 1.0 . For each pure compound critical pressure, the temperature and acentric factor $(\omega)$ as well as $P, Q, \varepsilon^{A B}$ and $\kappa^{A B}$ parameters are shown in Table 1 . The average absolute deviation (AAD) values for vapor pressure and saturated liquid molar volume are calculated using the equation

$$
A A D=\sum_{i=1}^{N p} \frac{\left|X_{\text {exp }, i}-X_{\mathrm{calc}, i}\right|}{X_{\text {exp }, i}} \frac{100}{N p}
$$

in which $N p$ is the number of experimental points of each pure compound and $X$ is related to each property (pressure or volume).

Using the $P$ and $Q$ parameters for each pure compound, and Eqs. (14) and (15), it is possible to calculate generalized adjustable parameters $\left(P_{1}\right.$ to $P_{3}$ and $Q_{1}$ to $\left.Q_{3}\right)$, whose values are shown in Table 2.

Table 2. Parameters of the Eqs. (14) and (15) for generalized alpha and beta as a function of acentric factor $(\omega)$.

\begin{tabular}{ccc}
\hline & & Eqs. (14) and (15) + proposed EOS \\
\hline \multirow{4}{*}{$\boldsymbol{P}(\omega)$} & $\boldsymbol{P}_{\boldsymbol{1}}$ & -0.9213 \\
& $\boldsymbol{P}_{2}$ & 2.9102 \\
& $\boldsymbol{P}_{3}$ & -0.1473 \\
& $\boldsymbol{Q}_{1}$ & 2.5101 \\
$\boldsymbol{Q}(\omega)$ & $\boldsymbol{Q}_{2}$ & -2.4298 \\
& $\boldsymbol{Q}_{3}$ & -4.2781 \\
\hline
\end{tabular}

It is important to emphasize that although the parameters presented in the Table 2 have been adjusted to the pure compounds presented in the Table 1, it is possible to apply these values for the correlations of the vapor pressure and densities for different alcohols which are not included in the parameterization procedure executed here.

Concerning the cubic part of the equations of state, the SAFT and CPA EOSs are simpler than the modified equation of state presented here. This is because each of these equations has one different set of adjustable parameters for correlation or prediction of the thermophysical properties of each pure compound.

According to (Kontogeorgios et al., 1996), it is possible to establish relations between the enthalpy of hydrogenbonding $\left(\Delta H^{\text {assoc }}\right)$ and $\left(\varepsilon^{A B}\right)$ as well as between the association volume $\left(\kappa^{A B}\right)$ and the hydrogen-bonding entropy $\left(\Delta S^{\text {assoc }}\right.$ ), using chemical and perturbation theories. The $\varepsilon^{A B}$ values obtained for each pure compound studied are within of the range 268 to $274 \mathrm{bar} \mathrm{L} \mathrm{mol}^{-1}$ (or 26.8 to $27.4 \mathrm{~kJ} \mathrm{~mol}^{-}$ ${ }^{1}$ ), given in (Kontogeorgios et al., 1996). These $\Delta H^{\text {assoc }}$ values pertain to the same intervals shown in (Kontogeorgios et al., 1996), (Koh et al., 1993), (Palombo et al., 2006), and Nath \& Bender, 1981) for alkanols and 
water. Satisfactory agreement is also observed for the $\kappa^{A B}$ values for the majority of the pure compounds shown in (Kontogeorgios et al., 1996). This information helps us to qualify as satisfactory the parameters generalization method proposed for the new associated equation of state.

With the critical pressure and critical temperature, acentric factor, generalized parameters $\left(P_{1}\right.$ to $\left.P_{3}\right)$ and $\left(Q_{1}\right.$ to $\left.Q_{3}\right)$, and characteristic parameters $\varepsilon^{A B}$ and $\kappa^{A B}$, it is possible to calculate the vapor pressure and liquid molar volume using eight adjustable parameters for each pure compound. If it is necessary to calculate the properties for all 15 pure compounds presented in the Table 1, 75 different parameters needs to be used. However, for this same quantity of pure compounds, the number of adjustable parameters can be only 8 , if common parameters $\left(P_{1}\right.$ to $\left.P_{3}\right)$, ( $Q_{1}$ to $Q_{3}$ ), presented in the Table 2, as well as the parameters $\varepsilon^{A B}=270$ bar L mol $^{-1}$ and $\kappa^{A B}=0.01$ have been employed in the Eqs. ((6) and (7)). In terms of deviations presented by the equations of state (SAFT, CPA, and Eqs. (6) and (7)), the values are approximately the same in both cases; this situation contributes to justify the use of a more physically consistent repulsive term, and the generalized functions for correlative or predictive calculations. (As a comparison, the original CPA EOS uses five adjustable parameters to calculate the pressure and molar volume properties for each pure compound; if the number of pure compounds is accounted 15 , the number of the adjustable parameters needed is 75 , such as if the non-generalization method is employed in the Eqs. (6) and (7).

In relation to the association term of the EOS, an important decision is necessary about choice of hydrogenbonding sites in the molecules. For the water molecule, (Perakis et al., 2006) took into account in their study for both three and four association sites while Huang and Radosz (Huang \& Radosz, 1990) took into account three association sites. For alkanols, two and three association sites were considered in (Huang \& Radosz, 1990), while three association sites were accounted for (von Solms et al., 2006) and (Queimada et al., 2005). Our decision is to adopt the three association sites for water molecules to facilitate the calculations, and contribute to making sense the suggestion presented in experimental studies (Wei et al., 1991): the possibility of the water molecules to form three association sites; for alkanols (Huang \& Radosz, 1990) and (von Solms et al., 2006) and (Queimada et al., 2005) show that three-site hydrogen-bonding scheme is more adequate than that represented by a two sites scheme. Just for information, it is possible to apply another association scheme for both water and alcohol molecules, as for example, 4 and 2, respectively. However, the improvement of the deviations is insignificant in comparison to those presented by the others EOSs.

In order to test the efficiency of the proposed EOS, the average absolute deviation (AAD) values between experimental and calculated data for vapor pressure and liquid molar volume of pure compounds were determined. The expression employed is identical to Eq. (21) and the values are shown in Table 3.

For vapor pressure data, it is possible to observe that the AAD values presented in Table 1 are smaller than those presented in the fourth column of Table 3 (original CPA EOS), except for 1-propanol and water. This comparison shows that the modifications proposed in the equation of state are capable of improving the correlation of the results, by using adjustable parameters specific to each pure compound (no employing generalization method). However, the calculated AAD values using the new generalized equation of state (second column of Table 3) are a little bit greater than those obtained in the original CPA EOS (fourth column) and original SAFT EOS (sixth column); the reason of this characteristic can be attributed to the generalization of the adjustable parameters, since the calculations are executed accounting for all pure compounds. Comparisons between the AAD values shown in Table 1 with those presented in the sixth column of the Table 3 shows that the calculated vapor pressure values are improved by use of the proposed EOS (again, without employing the generalization method).

Table 3. AAD between experimental and calculated vapor pressure and liquid molar volume.

\begin{tabular}{|c|c|c|c|c|c|c|}
\hline \multirow[b]{2}{*}{ Compound } & \multicolumn{2}{|c|}{$\begin{array}{c}\text { Proposed EOS for } \\
\text { generalized alpha and beta } \\
\text { functions }\end{array}$} & \multicolumn{2}{|c|}{$\begin{array}{c}\text { Original } \\
\text { CPA EOS (Kontogeorgios et } \\
\text { al., 1996) } \\
\end{array}$} & \multicolumn{2}{|c|}{$\begin{array}{c}\text { Original } \\
\text { SAFT EOS (Huang \& Radosz, } \\
\text { 1990) }\end{array}$} \\
\hline & $\Delta P(\%)$ & $\Delta V(\%)$ & $\Delta P(\%)$ & $\Delta V(\%)$ & $\Delta P(\%)$ & $\Delta V(\%)$ \\
\hline Methanol & 1.53 & 2.19 & 0.84 & 0.57 & 0.83 & 0.88 \\
\hline Ethanol & 1.03 & 1.67 & 0.63 & 0.29 & 0.86 & 0.83 \\
\hline 1-propanol & 0.87 & 2.26 & 0.33 & 0.47 & 0.16 & 1.20 \\
\hline 2-propanol & 1.05 & 3.52 & --- & --- & 0.27 & 0.96 \\
\hline 1-butanol & 0.63 & 2.83 & 0.76 & 0.79 & 0.23 & 1.00 \\
\hline 2-butanol & 0.81 & 2.80 & --- & --- & 0.32 & 1.20 \\
\hline 2-methyl-1-propanol & 1.19 & 3.11 & --- & --- & 0.58 & 1.40 \\
\hline 2-methyl-2-propanol & 1.30 & 1.97 & --- & --- & 0.21 & 0.79 \\
\hline 1-pentanol & 1.24 & 2.88 & 0.42 & 2.54 & 0.32 & 1.10 \\
\hline 1-hexanol & 0.29 & 3.08 & 0.34 & 0.90 & 0.77 & 1.22 \\
\hline 1-heptanol & 0.30 & 2.39 & --- & --- & 0.61 & 0.96 \\
\hline 1-octanol & 1.52 & 3.05 & 2.16 & 2.51 & 1.00 & 1.00 \\
\hline 1-nonanol & 0.53 & 2.18 & 1.82 & 2.38 & 1.10 & 0.47 \\
\hline 1-decanol & 1.46 & 3.16 & 1.46 & 0.80 & 2.10 & 0.57 \\
\hline Water & 1.66 & 3.90 & 0.42 & 0.94 & 1.30 & 3.20 \\
\hline OVERALL DEVIATION (OD) & 0.98 & 2.67 & 0.61 & 0.81 & 0.71 & 1.12 \\
\hline
\end{tabular}


Considering the experimental uncertainties presented in the references (Beaton et al., 1989 a and b) and (Vargaftik, 1975), calculated values of the AAD for vapor pressure and liquid molar volumes are $0.89 \%$ and $1.02 \%$, respectively, considering non-generalized version of the proposed EOS; for generalized version, where common adjustable parameters are used, calculated values of the AAD for studied properties are $1.14 \%$ (vapor pressure) and $1.19 \%$ (liquid molar volume).

Both saturated liquid and vapor molar volumes are obtained by solution of Eq. (6) and six roots are obtained: one negative, two complex numbers, and three real. Considering the three real roots, the smallest one corresponds to the calculated liquid molar volume while the largest one corresponds to calculated vapor molar volume value. In Tables 1 and 3 the AAD values for the saturated liquid molar volume are shown. The AAD values presented in Table 1 (last column) are smaller than those presented in the fifth and seventh columns of the Table 3, confirming the efficiency of the new equation of state. As the adjustable parameters are obtained by generalization of all pure compounds, the AAD values calculated using the generalized new equation of state, are greater than those presented in the original CPA and SAFT EOS, due to the same reason mentioned above.

The improvement of the AAD values can be attributed to the quantitative capability of the Peng-Robinson EOS to predict and correlate pure compounds equilibrium properties as well as to the adequate choice of generalized functions to calculate these properties (Coquelet et al., 2004). Another contribution to deviation improvements can be associated with the theoretical basis applied by Carnahan-Starling (Carnahan \& Starling, 1969 \& 1972) in the development of the repulsive term of the equation of state; this characteristic is also observed in several works shown in the literature (De Santis et al., 1976) and (Zhong \& Masuoka, 1997).

The overall deviation (OD) values presented in Table 1 for vapor pressure and liquid molar volume are smaller than the OD values calculated for both properties using the original SAFT EOS (Huang \& Radosz, 1990) accounting for the same pure compounds studied in the present work. However, in the original CPA EOS work (Kontogeorgios et al., 1996), only ten pure compounds (out of 15 studied here) are included in the OD calculations. This difference between numbers of compounds can explain the smaller value of OD for vapor pressure $(0.612 \%)$ in relation to the OD value given in Table $1(0.691 \%)$, although the OD value from Table $1(0.735 \%)$ for liquid molar volume is greater than that presented in Table $3(0.813 \%)$. Both comparisons imply that the efficiency improvement of property calculations is due to the proposed modifications in the equation of state developed.

Considering Table 3, if comparisons are made between the OD values in the second and third columns $(0.98 \%$ and $2.67 \%$ ) with those shown in the fourth and fifth columns $(0.61 \%$ and $0.81 \%)$, and those shown in the sixth and seventh columns $(0.71 \%$ and $1.12 \%)$, it is obvious that the first group values are greater than the last two groups (as in the case with the AAD values). In spite of this increase in OD values, it is possible to observe that the proposed generalized equation of state is superior, since only one group of adjustable parameters is necessary to correlate the vapor pressure and liquid molar volume data of various pure compounds. Another fact that probably contributes to better AAD and OD values is the $T r$ range considered in the present work $(0.50<T r \leq 1.00)$, while in the original CPA and SAFT EOS its values are $(0.50<T r<0.90)$ and $(0.50<$ $\operatorname{Tr}<0.95)$, respectively. It is well known that various equations of state are inadequate for representation of the pressure and molar volume properties in the proximity of the critical condition of pure compounds. However, the proposed equation of state is able to represent the vapor pressure and liquid molar volume data (and also for vapor molar volume data, although the results are not presented in this work) in the critical region.

\section{Conclusions}

Modifications of the CPA equation of state (Kontogeorgios et al., 1996) consisting of replacing its original repulsive term by that proposed by CarnahanStarling (Carnahan \& Starling, 1969 \& 1972), and considering the attractive and repulsive parameters to be temperature dependent, are proposed. The calculated values of the vapor pressure, and vapor and liquid molar volumes are in satisfactory agreement with the experimental data of these properties for alkanols and water compounds, within of the reduced temperature range $0.50<T r \leq 1.00$. This $T r$ range was chosen since the chemical industry works with temperatures and pressures which are, in most cases, lower than those related to the critical condition (below $T r=$ $1.00)$, especially for processes involving the alkanols and water. It is important to emphasize that the proposed EOS was applied only to the pure compounds in the present study. Extensions of the application to mixtures containing alkanols + water are being analyzed and will be presented in the near future.

The generalization of the energetic and co-volume adjustable parameters is advantageous since it is possible to use only one set of parameters to calculate the pure compounds properties; besides, these same parameters set can be used to correlate the properties of different alcohols other than those studied here.

The AAD values show that the predictive capability of the proposed EOS is better than those shown in the literature (Huang \& Radosz, 1990) and (Kontogerorgis et al., 1996) for the majority of the studied pure compounds. This improvement is probably due to the efficiency of the Peng-Robinson equation of state in representing the pressure and molar volume properties more adequately than the Soave-Redlich-Kwong EOS (Soave, 1972), and due to the theoretical consistency of the repulsive term employed in the Eq. (6). In spite of the increase in OD value, compared to original CPA and SAFT EOS (Table 3) the results obtained with the proposed equation of state are physically consistent. The calculations show that the introduction of beta functions and common values of energy and volume of association $\left(\varepsilon^{A B}\right.$ and $\left.\kappa^{A B}\right)$ for alkanols and water contributes to facilitate the calculation routines and improves the correlative capability of the EOS.

\section{Nomenclature}

$\mathrm{AAD}=$ Average Absolute Deviation (\%)

$a=$ Attraction parameter (bar $\mathrm{L}^{2} \mathrm{~mol}^{-2}$ )

$b=$ Co-volume parameter $\left(\mathrm{L} \mathrm{mol}^{-1}\right)$

$g(\rho)=$ Radial distribution function

$N p=$ Number of experimental data

$O D=$ Overall deviation $(\%)$

$p=$ Pressure (bar)

$R=$ Universal constant of gases $\left(\right.$ bar $\mathrm{L} \mathrm{mol}^{-1} \mathrm{~K}^{-1}$ ) 
$T=$ Temperature $(\mathrm{K})$

$\operatorname{Tr}=$ Reduced temperature

$X_{A}=$ Site fraction not bonded at site A

$X_{B}=$ Site fraction not bonded at site B

$V=$ Molar volume $\left(\mathrm{cm}^{3} \mathrm{~mol}^{-1}\right)$

$\mathrm{Z}=$ Compressibility factor

\section{Greek Letters}

$\alpha=$ alpha function

$\beta=$ beta function

$\Delta^{A B}=$ association strength

$\varepsilon^{A B}=$ energy of association (bar $\mathrm{L} \mathrm{mol}^{-1}$ )

$\kappa^{A B}=$ volume of association

$\eta=$ packing fraction of hard spheres

$\rho=$ molar density $\left(\mathrm{mol} \mathrm{cm}{ }^{-3}\right)$

$\omega=$ acentric factor

\section{Subscripts}

$\mathrm{c}=$ critical condition

calc $=$ calculated property

$\exp =$ experimental property

1,2,3 = specific of each $P$ and $Q$ adjustable parameters of Eqs. (15) and (16).

\section{References}

Abbot, M. M. (1989). Thirteen Ways of Looking at the van der Waals Equation, Chemical Engineering Progress, (2), 25-37.

Beaton, C. F., Ambrose, D., Brunner, E., Chase, M. W., Downey, J. R., Hobson G., Humphreys, A. E., Jamieson, D. T., Knight, S. R., Schoenberg, M., Walton, J., (1989). Ortobaric densities and molar volumes of liquids, Alcohols, Engineering Sciences Data Unit ESDU, Eng. Sci. Data Item Nr. 89037.

Beaton, C. F., Ambrose, D., Foxcroft, H. J., Hobson, G., Jamieson, D. T., Knight, S. R., Rowell, G. M., Schoenberg, M., White, Jr., H. J., (1989). Vapor pressures and critical points of liquids, Alcohols, Engineering Sciences Data Unit - ESDU, Eng. Sci. Data Item Nr. 89028 Vapor Pressure Data.

Carnahan, N. F., Starling, K. E., (1969). Equation of State for Nonattracting Rigid Spheres. The Journal of Chemical Physics, 51, 635-636.

Carnahan, N. F., Starling, K. E., (1972). Intermolecular Repulsions and the Equation of State for Fluids. AIChE. J., 18, 1184-1189.

Coquelet, C., Chapoy, A. Richon, D., (2004). Development of a New Alpha Function for the Peng-Robinson Equation of State: Comparative Study of Alpha Function Models for Pure Gases (Natural Gas Components) and Water-Gas Systems. Int. J. Thermophys. 25, 133-158.

De Santis, R., Gironi, F., Marrelli, L., (1976). Vapor-Liquid Equilibrium from a Hard-Sphere Equation of State. Ind. Eng. Chem. Fundam., 15, 183-189.

Fuller, G.G., (1976). A Modified Redlich-Kwong-Soave Equation of State Capable of Representing the Liquid State. Ind. Eng. Chem. Fundam., 15, 254-257.

Haghtalab, A., Mahmoodi, P., Mazloumi, S. H., (2011). A modified Peng-Robinson equation of state for phase equilibrium calculation of liquefied, synthetic natural gas, and gas condensate mixtures. Can. J. of Chem. Eng., 89, 1376-1387.

Hamam, S. E. M., Chung, W. K., Elshayal, I. M, Lu, B. CY., (1977). Generalized Temperature-Dependent Parameters of the Redlich-Kwong Equation of State for Vapor-Liquid Equilibrium Calculations. Ind. Eng. Chem. Process Des. Dev., 16, 51-59.

Huang, S. H., Radosz, M., (1990). Equation of State for Small, Large, Polydisperse, and Associating Molecules. Ind. Eng. Chem. Res., 29, 2284-2294.

Koh, C. A., Tanaka, H., Walsh, J. M., Cubbins, K. E., Zollweg, J. A., (1993). Thermodynamic and structural properties of methanol-water mixtures: experiment, theory and molecular simulation. Fluid Phase Equilibria, 83, 51-58.

Kontogeorgis, G. M., Michelsen, M. L., Folas, G. K., Derawi, S., von Solms, N., Stenby, E. H., (2006). Ten Years with the CPA (Cubic-Plus-Association) Equation of State. Part 1. Pure Compounds and Self-Associating Systems. Ind. Eng. Chem. Res., 45, 4855-4868.

Kontogeorgis, G. M., Voutsas, E. C., Yakoumis, I. V., Tassios, D. P., (1996). An Equation of State for Associating Fluids, Ind. Eng. Chem. Res., 35, 43104318.

Kontogeorgis, G. M., Yakoumis, I. V., Meijer, H., Hendriks, E. M., Moorwood, T., (1999). Multicomponent phase equilibrium calculations for water-methanol-alkane mixtures. Fluid Phase Equilibria, 158, 201-209.

Kuester, J. L., Mize, J. H., (1973). Optimization Techniques, McGraw-Hill, USA.

Kutney, M.C., Dodd, V.S., Smith, K.A., Herzog, H.J., Tester, J.W., (1997). A hard-sphere volume translated van der Waals equation of state for supercritical process modeling 1. Pure components. Fluid Phase Equilibria, $128,149-171$.

Mathias, P. M., Copeman, T. W., (1983). Extension of the Peng-Robinson Equations of State to Complex Mixtures: Evaluation of the Various Forms of the Local Composition Concept. Fluid Phase Equilibria, 13, 91108.

Nasrifar, K., Moshfeghian, M., (2001). A new cubic equation of state for simple fluids: pure and mixture. Fluid Phase Equilibria, 190, 73-88.

Nath, A., Bender, E., (1981). On the thermodynamics of associated solutions. I. An analytical method for determining the enyhalpy and entropy of association and equilibrium constant for pure liquid substances. Fluid Phase Equilibria, 7, 275-287.

Sassi, P., Paolantoni, M., Morresi, A., Cataliotti, R. S., (2006). Comparison of Hydrogen Bonding in 1-Octanol and 2-Octanol as Probed by Spectroscopic Techniques. J. Phys. Chem. B, 110, 18017-18025.

Peng, D-Y, Robinson, D.B., (1976). A New Two-Constant Equation of State. Ind. Eng. Chem. Fundam., 15, 59-64.

Perakis, C. A., Voutsas, E. C., Magoulas, K. G., Tassios, D. P., (2007). Thermodynamic Modeling of the Water + 
Acetic Acid $+\mathrm{CO}_{2}$ System: The Importance of the Number of Association Sites of Water and of the Nonassociation Contribution for the CPA and SAFTType Models. Ind. Eng. Chem. Res., 46, 932-938.

Queimada, A. J., Miqueu, C., Marrucho, I. M., Kontogeorgis, G. M., Coutinho, J. A. P., (2005). Modeling vapor-liquid interfaces with the gradient theory in combination with the CPA equation of state. Fluid Phase Equilibria, 228, 479-485.

Ravagnani, S.P., D’Avila, S.G., (1985). VLE of Polar Mixtures: A New Generalized Correlation. Proceedings of IV International Chemical Engineering Conference, CHEMPOR'85, Coimbra, Portugal, 05 / 01 - 05.

Rosenbrock, H. H., (1960). An Automatic Method for Finding the Greatest or Least Value of a Function, Computer Journal, 3, 175-184.

Sadus, R. J., (2001). Equations of state for fluids: The Dieterici approach revisited. J. Chem. Phys., 115, 14601462.

Soave, G., (1972). Equilibrium constants from a modified Redlich-Kwong equation of state. Chem. Eng. Sci., 27, 1197-1203.

Toghiani, H., Viswanath, D.S., (1986). A Cubic Equation of State for Polar and Apolar Fluids. Ind. Eng. Chem. Process Des. Dev., 25, 531-536.

Trebble, M.A., Bishnoi, P.R., (1987). Development of a new four-parameter cubic equation of state. Fluid Phase Equilibria 35, 1-18.

Vargaftik, N.B., (1975). Handbook of Physical Properties of Liquid and Gases (Pure Substances and Mixtures), $2^{\text {nd }}$ Edition, John Willey, D.C.

von Solms, N., Michelsen, M. L., Passos, C. P., Derawi, S. O., Kontogeorgis, G. M., (2006). Investigating Models for Associating Fluids Using Spectroscopy. Ind. Eng. Chem. Res., 45, 5368-5374.

Wei, S., Shi, Z., Castleman, A., (1991). Mixed Cluster Ions as a Structure Probe: Experimental Evidence for
Clathrate Structure of $\left(\mathrm{H}_{2} \mathrm{O}\right)_{20} \mathrm{H}^{+}$and $\left(\mathrm{H}_{2} \mathrm{O}\right)_{21} \mathrm{H}^{+}$. J. Chem. Phys., 94, 3268-3270.

Wei, Y.S., Sadus, R.J., (2000). Equations of State for Calculation of Fluid-Phase Equilibria. AIChE J., 46, 169-196.

Wei, Y.S., Sadus, R.J., Franck, E.U., (1996). Binary mixtures of water + five noble gases: comparison of bimodal and critical curves at high pressures. Fluid Phase Equilibria 123, 1-15.

Wertheim, M. S., (1984a) Fluids with Highly Directional Attractive Forces. I. Statistical Thermodynamics. J. Stat. Phys., 35, 19-34.

Wertheim, M. S., (1984b). Fluids with Highly Directional Attractive Forces. II. Thermodynamic Perturbation Theory and Integral Equations. J. Stat. Phys., 35, 35-47.

Wertheim, M. S., (1986a). Fluids with Highly Directional Attractive Forces. III. Multiple Attraction Sites. J. Stat. Phys., 42, 459-476.

Wertheim, M. S., (1986b). Fluids with Highly Directional Attractive Forces. IV. Equilibrium Polimerization, $J$. Stat. Phys., 42, 477-492.

Wertheim, M. S., (1986c). Fluids of Dimerizing Hard Spheres and Mixtures of Hard Spheres and Dispheres. $J$. Chem. Phys., 85, 2929-2936.

Wertheim, M. S., (1987). Thermodynamic Perturbation Theory of Polymerization, J. Chem. Phys., 87, 73237331.

$\mathrm{Xu}, \mathrm{Z}$, Sandler, S.I., (1987). Temperature dependent parameters and Peng-Robinson equation of state. Ind. Eng. Chem. Res., 26, 601-606.

Zhong, C., Masuoka, H., (1997). An EOS/G ${ }^{\mathrm{E}}$ type mixing rule for perturbed hard sphere equation of state and its application to calculation of solid solubility in supercritical carbon dioxide. Fluid Phase Equilibria, $141,13-23$ 